\title{
Role of Magnesium in Abdominal wall Dyskinesia.
}

\footnotetext{
1. MBBS, DA, FCPS,

M.Sc. (Pain Medicine)

Professor Anesthesiology Pain and Intensive Care

Sahara Medical College, Narowal.
}

Correspondence Address:

Dr. Haq Dad Durrani

Department of Anesthesiology Pain and Intensive Care

Sahara Medical College, Narowal.

drhaqdad@szmc.edu.pk

profhaqdad@gmail.com

Article received on:

06/07/2020

Accepted for publication:

$14 / 09 / 2020$

\begin{abstract}
Haq Dad Durrani'
ABSTRACT... Objective: Jerky involuntary intermittent abdominal wall movements are mentioned with numerous names including abdominal wall dyskinasia and belly dancer's dyskinesia. Literature incorporates a sizeable amount of case reports. Patterson analyzed the largest series published in 2011. Although associated with central and peripheral nervous system disorders and published precipitating factors like anxiety, operations particularly abdominal, diarrhea, nutritional imbalances, pregnancy under the umbrella of idiopathic/ psychogenic /functional belly's dancer's dyskinesia. Various investigations including blood count, ESR, CT, MRI, ECG plain radiography do not reflect any abnormality. Similarly a large number of modalities are utilized to manage the disorder from diazepam to phrenic nerve block; however no definite treatment has been mentioned. Magnesium calms down the excitability of central and peripheral nervous systems by inhibition of NMDA receptors and calcium influx mediated acetylcholine release. Magnesium deficiency results in excitability of the excitable tissues resulting in neuromuscular hyperactivity. We are reporting a patient who presented with abdominal wall dyskinasia, managed successfully with an infusion of magnesium sulphate which has never been described.
\end{abstract}

Key words: Abdominal Wall Dyskinasia, Belly Dancer's Dyskinesia, Jerky Abdominal Movements, Magnesium Deficiency.

Article Citation: Durrani HD. Role of magnesium in abdominal wall dyskinesia. Professional Med J 2021; 28(2):267-270.

https://doi.org/10.29309/TPMJ/2021.28.02.5468

\section{INTRODUCTION}

Belly dancer's dyskinesia usually presents as visible or non visible repetitive involuntary rolling or fluttering of abdominal wall or epigastrium. The rate of rolling is variable ranging from 35 to 480 per minute. Fluttering may be so intense that bed on which patient lying may shake. Myoclonus is considered responsible for this condition. Various case reports have mentioned different respiratory patterns. There may be tachypnea, diaphragmatic flutter and apneustic respiration. Respiratory pattern may be dual; a normal respiratory pattern is superimposed by high frequency fluttering respiration. Breath holding or distraction cannot modify the fluttering respiration. The fluttering may be unilateral but usually involves abdominal wall bilaterally. In most of cases, fluttering is not noticed by family during sleep of patient. Heart rate and blood gas analysis are usually within normal range. The condition may have isolated or chronic recurrent presentation lasting for minutes or hours. ${ }^{1}$ In various case reports it is also termed as abdominal wall dyskinesia, intermittent bursts of abdominal jerky movements or diaphragmatic flutter. ${ }^{2,3}$ Antony van Leeuwenhoek was the first person who described diaphragmatic flutter or belly dancer's syndrome in 1723. ${ }^{4,5}$ Among others Victoria Patterson published a largest case series in $2011 .^{1}$

The Pathophysiology of this syndrome has not yet been understood. Multiple causations has been proposed including disorders of Central and/ or peripheral nervous systems e.g, encephalitis, irritation of phrenic nerve, mediastinal, pleural, pulmonary, cardiac, intra abdominal disorders, cervical spine trauma, operations of thoracic or abdominal cavity, diarrhea, pregnancy and vaginal delivery. ${ }^{1,2,6,7,8}$ In significant number of patients, exact cause of these movements could not be 
identified, hence in these patient it is considered as idiopathic, psychogenic or functional in origin. ${ }^{3}$ Although a large number of precipitating factors could be considered, however no single cause of these movements has yet been documented in literature. ${ }^{6}$

It has been observed that almost all of the cases of idiopathic belly dancer's dyskinesia are preceded by a period of anxiety, abdominal disorders (operations, diarrhea, malabsorption) and use of aminoglycides especially gentamicin. It also has been found to be more common in patient with Type II diabetes mellitus, pregnancy or who are currently taking diuretics. ${ }^{1-6}$

Various treatment modalities including diazepam, carbamazepine, clonazepam, chlorpromazine, haloperidol, Entonox, botulinum toxins injections into rectus abdominus/ external oblique muscles, and phrenic nerve block have been tried with varying degree of success, but there has been no consensus about best treatment modality for management of this dyskinesia till date. ${ }^{1,2}$

G. Grases found that significant anxiety increases urinary excretion and causes partial depletion of magnesium. ${ }^{9}$ Additionally, inflammation and thickening of bowel walls as seen in crohn's disease can reduce absorption of magnesium leading to deficiency of magnesium. ${ }^{10}$

In pregnancy hypomagnesaemia has been observed in $57.2 \%$ of cases which has been proposed to be due to several fold increase in estrogen level which increases utilization of magnesium. ${ }^{11,12}$

It has been proposed that activation of $\mathrm{N}$ methyl $D$ aspartate receptors leads to massive depolarization of neurons resulting in bursts of action potentials. Magnesium prevents burst of these action potentials by antagonism of NMDA receptors. Moreover, Influx of $\mathrm{Ca}^{++}$causes release of acetylcholine from storage vesicles resulting in depolarization and action potential at motor end plate. Magnesium is voltage dependent calcium channel antagonist so inhibits acetylcholine release, membrane depolarization and action potential generation at neuromuscular junction of striated muscles. Finally, Inhibition of alpha and gamma motor neurons by GABA ergic receptors is potentiated by magnesium. ${ }^{13,14}$ Neuromuscular irritability, CNS hyper excitability and cardiac arrhythmias are the most important presentation of magnesium deficiency. ${ }^{13}$ Magnesium deficiency results in increased acetylcholine release and neuromuscular excitation and hyperactivity of alpha and gamma motor neuron. ${ }^{14}$

The clinical features of magnesium deficiency are primarily neurological and neuromuscular including neuromuscular hyperactivity, tremors, fasciculation, psychiatric disturbances, personality changes, Trousseau sign etc which reverted to normal following correction of magnesium deficiency. ${ }^{15}$

Commonly reported range of serum magnesium is $1.7-2.4 \mathrm{mg} / \mathrm{dl}$. Serum magnesium level may be normal in patients deficient in magnesium. Magnesium deficiency is under diagnosed by considering normal level as standard. Although unambiguous test for magnesium deficiency is not available, yet among the available laboratory facilities, magnesium loading test is considered Gold Standard Test. ${ }^{16}$

Based on previous observations, we used intravenous magnesium infusion in one of patient who presented with Belly dancer's dyskinesia and are presenting its results in this case report.

\section{CASE REPORT}

A 21 years old woman presented with acute abdominal pain and was diagnosed as acute appendicitis. She was treated conservatively with intravenous antibiotics including Gentamicin for 5 days by our surgical colleagues and was discharged. After four weeks of discharge, she presented again with recurrence of symptoms of acute appendicitis. She underwent appendectomy under general anesthesia with perioperative cover of intravenous Gentamicin, Cephradine and Flagyl without any complication.

Two weeks after appendectomy, she developed 
stridor, rapid deep breathing, twitching of abdominal muscles with intense movements. These movements persisted despite breath holding and distraction. Rapid and deep breathing led to abdominal distension as a result of aerophagia i.e, excessive air swallowing. She also had paroxysmal weakness of muscles, disturbed gait and history of fall off and on.

She was referred to a consultant psychiatrist to consider psychogenic cause of these movements. She was diagnosed as conversion disorder and was started on an SSRI, Sertraline. There was no improvement in paroxysmal twitching after 10 weeks of treatment with Sertraline which continued to occur 3 to 4 times a day, persisting for more than an hour at a time. It was associated with pain, limb weakness, disturbed gait and falls.

Patient's serum Calcium was $9.5 \mathrm{mg} / \mathrm{dl}$ and Serum magnesium was $1.7 \mathrm{mg} / \mathrm{dl}$ (Normal range 1.7 $2.4 \mathrm{mg} / \mathrm{dl})$.

As it is known in the literature that patient can have magnesium deficiency despite normal serum magnesium level, therefore we proceeded with intravenous infusion of $4 \mathrm{~g}$ of magnesium sulphate in $1 \mathrm{~L}$ of Ringer's lactate over 4 hours on the day 1 -, reducing it down to 2 grams of magnesium sulphate in Ringer's lactate over 4 hours on day 2. We documented a dramatic recovery in patient's symptoms. Patient was advised to increase intake of magnesium containing diet including green leafy vegetables, almonds and nuts. We have come to know vide follow up that she is asymptomatic for the last 12 years.

\section{DISCUSSION}

We found that intravenous replacement of magnesium resolved symptoms of Belly dancer's dyskinesia in our patient who had normal serum magnesium level. We propose that our patient had reduced total body magnesium contents despite normal serum magnesium levels which was due to reduced daily intake of magnesium secondary to use of processed food her selection of type of vegetables/fruits, subsequent Inflammation of bowel wall at ileocecal region resulted in reduced absorption of magnesium leading to low total body magnesium contents Additionally, administration of Gentamicin at the time of conservative management and later on in perioperative period further worsened magnesium deficiency by increasing renal excretion ${ }^{17}$ resulting in belly dancer's dyskinesia.

Previous studies have suggested that magnesium replacement improves symptoms of various psychiatric and neurological illnesses including depression and Belly dancer's dyskinesia. ${ }^{18}$ Osama S M Amin et al has reported an improvement in Belly dancer's dyskinesia after administration of Diazepam. ${ }^{3}$ However, in another review, clonazepam, Gabapentine, and Botulinium injections have been proven only partially effective in providing relief in Belly dancer's dyskinesia. ${ }^{1}$ Our patient also did not improve with S.S.R.I. treatment and instead responded to administration of magnesium infusion. Most of the cases reported as idiopathic belly dancer's dyskinesia occurred/followed either malabsorption, ${ }^{1}$ diarrhea ${ }^{2,6}$ kleen enema, ${ }^{3}$ or pregnancy ${ }^{7,8}$ all potential causes of magnesium deficiency. ${ }^{10,11,12,19}$

We suggest that in patients with abdominal wall dyskinesia, serum magnesium should be checked and a magnesium loading test should be tried to identify total body magnesium deficiency as a cause of Idiopathic abdominal wall dyskinesia. Our case presentation suggests that intravenous magnesium infusion is a potentially effective treatment in patient with abdominal wall dyskinesia.

\section{Copyright $@ 14$ Sep, 2020.}

\section{REFERENCES}

1. Patterson V. Belly dancer's syndrome: Causes, clinical presentations, and treatment. Available from: http:// www.logan.edu/mm/files/LRC/Senior-Research/2011Dec-31.pdf. [Last accessed on 2020 April18 ]

2. Ashutosh Gupta SK. Belly Dancer's Dyskinesia: A Glimpse of a Rare Phenomenon. Cureus. 2017 Jul; 9(7).

3. Amin OS, Abdulkarim QH, Shaikhani M. Intermittent bursts of abdominal wall jerky movements: belly dancer's syndrome?. Case Reports. 2012 Dec 24; 2012:bcr2012007393. 
4. Moreira AR, Freitas AC, Rocha F, Cardoso R, Fonseca P. Síndrome de Belly Dancer:- A diagnostic challenge. Nascer e Crescer. 2017 Mar; 26(1):49-52.

5. Larner AJ. Antony van Leeuwenhoek and the description of diaphragmatic flutter (respiratory myoclonus). Movement disorders: official journal of the Movement Disorder Society. 2005 Aug; 20(8):917-8.

6. Rathore C, Prakash S, Bhalodiya D. Belly dancer's dyskinesia: A rare movement disorder. Neurology India. 2018 Mar 1; 66(7):156-7

7. Aldabbour B, E'Leimat I, Alhayek K, Momani A. Recurrent Belly Dancer's Dyskinesia with Pregnancy. Journal of movement disorders. 2019 May 1;12(2):1289.

8. Herbert J, Hassanaien M. Case Study: A Bellydancing Womb. In Royal College of Obstetricians and Gynaecologists World Congress 2014 Mar 30

9. Grases G, Pérez-Castelló JA, Sanchis P, Casero A, Perelló J, Isern B, Rigo E, Grases F. Anxiety and stress among science students. Study of calcium and magnesium alterations. Magnesium research. 2006 Jun $1 ; 19(2): 102-6$.

10. Long $\mathrm{S}$, Romani AM. Role of cellular magnesium in human diseases. Austin journal of nutrition and food sciences. 2014 Nov 18; 2(10).

11. Workinger JL, Doyle R, Bortz J. Challenges in the diagnosis of magnesium status. Nutrients. 2018 Sep; 10(9):1202.
12. Eltayeb R, Rayis DA, Sharif ME, Ahmed AB, Elhardello $\mathrm{O}$, Adam I. The prevalence of serum magnesium and iron deficiency anaemia among Sudanese women in early pregnancy: A cross-sectional study. Transactions of the royal society of tropical medicine and hygiene. 2019 Jan 1; 113(1):31-5.

13. Nardone R, Brigo F, Trinka E. Acute symptomatic seizures caused by electrolyte disturbances. Journal of clinical neurology. 2016 Jan 1; 12(1):21-33.

14. Antonenko SA. Influence of magnesium on excitability of neurons of different levels of their organization. Journal of Education, Health and Sport. 2019 Nov 30; 9(11):314-22.

15. World Health Organization. Nutrients in drinking water. World Health Organization; 2005.

16. Ismail AA, Ismail NA. Magnesium: A mineral essential for health yet generally underestimated or even ignored. J. Nutr. Food Sci. 2016; 6(2).

17. Nanji AA, Denegri JF. Hypomagnesemia associated with gentamicin therapy. Drug intelligence \& clinical pharmacy. 1984 Jul; 18(7-8):596-8.

18. Derom ML, Sayón-Orea C, Martínez-Ortega JM, Martínez-González MA. Magnesium and depression: a systematic review. Nutritional neuroscience. 2013 Sep $1 ; 16(5): 191-206$.

19. GuthmanR. Whatarethecauses of hypomagnesaemia? Evidence Based Answer. The Journal of Family practice. 2005 Feb; 54(2):174-6.

\begin{tabular}{|l|l|l|}
\hline \multicolumn{2}{|c|}{ AUTHORSHIP AND CONTRIBUTION DECLARATION } \\
\hline Sr. \# & Author(s) Full Name & \multicolumn{1}{c|}{ Contribution to the paper } \\
\hline 1 & Haq Dad Durrani & $\begin{array}{l}\text { Considered the administration of } \\
\text { magnesium to suppress exaggerated } \\
\text { neuromuscular excitibility, Searched } \\
\text { the literature which reflected a large } \\
\text { number of patients having magnesium } \\
\text { deficiency might have normal serum } \\
\text { magnesium and attending psychiatry } \\
\text { and medicine O.P. Administered } \\
\text { magnesium infusion, Followed the } \\
\text { patient, Again searched the literature, } \\
\text { Manuscript writing, Checked plagiarism. }\end{array}$ \\
\hline
\end{tabular}

